\title{
Time-Resolved Electron Holography by Interference Gating
}

Tore Niermann ${ }^{1}$, Tolga Wagner ${ }^{1}$ and Michael Lehmann ${ }^{1}$.

1. Technische Universität Berlin, Institut für Optik und Atomare Physik, Berlin, Germany

Off-axis electron holography is an established technique for quantitative measurement of electric and magnetic fields. Due to its high sensitivity to the coherency of the electron beam, it requires extended exposure times (typically several seconds) to achieve a sufficient signal-to-noise ratio. We present a simple, yet promising, method for holographic measurements of periodic reversible processes with temporal resolutions in the microsecond regime by means of a gating approach.

When the holographic setup is disturbed by temporal variations, the interference pattern is destroyed. The measurement idea is to exploits this sensitivity, by deliberately introducing these disturbances, except for short fractions of the periodic process. Only during these short time intervals, when the setup is kept stable, an interference pattern is formed. By changing the delay of these stable intervals with respect to the periodic process, the whole periodic process can be temporally sampled. We call this method interference gating [1].

We tested this method using two different approaches. The first approach is to change the voltage of the electron optical biprism, thus changing the angle of overlap in the hologram. While successful in principle, this approach has the drawback, that the central part of the interference pattern is not disturbed, hence only the outer parts of the pattern become time resolved. The second approach is to introduce small beam deflections above the objective lens. This is readily achieved by applying small currents to the scanning coils present in nearly every microscope. Due to non-symmetric lens aberrations this causes a different phase shift in both partial waves. Figure 1 shows several periods of the current applied to these coils plotted on top of each other. Only during the small interval in the center, the beam is deflected to equal amounts in each period, thus only during these times a stable interference pattern is formed.

As test specimen a severed connection on a DENS heating chip is used (Figure 2). The projected potential across this connection is measured, which is proportional to the externally applied voltage. Externally a square wave voltage is applied with $1 \mathrm{kHz}$ repetition frequency. Figure 3 shows the phases of two reconstructed holograms. The reconstructed hologram on the left hand side is obtained without interference gating. It shows the $+/-\pi$ phases typical for double exposure holography [2], as here both levels of the applied voltage contribute to equal amounts.

In contrast, the phase of the reconstructed hologram on the right hand side is obtained with interference gating by beam deflection. With the gating signal applied only stable interference patterns are formed in the indicated interval, i.e. during an applied positive voltage. Consequently, only a slope within the phase signal corresponding to the electrical field present between the contacts is observed. This clearly demonstrates the feasibility of the interference gating approach, here with a time resolution of $50 \mu \mathrm{s}$.

\section{References:}

[1] T. Niermann, M. Lehmann and T. Wagner, Ultramicroscopy 182 (2017), p. 54.

[2] V. Migunov et al, Ultramicroscopy 178 (2017), p. 48. 


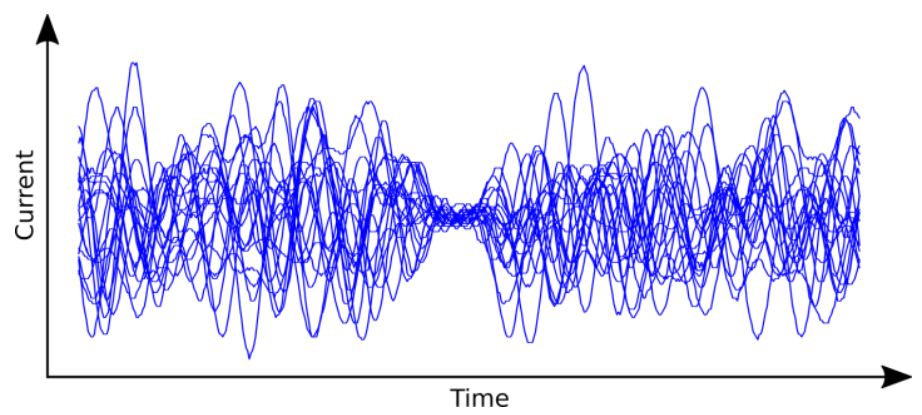

Figure 1. Several periods of current signal applied to the scan coils of the microscope (measured by oscilloscope). During the central time interval a constant current is applied. During other times a white noise signal is applied.

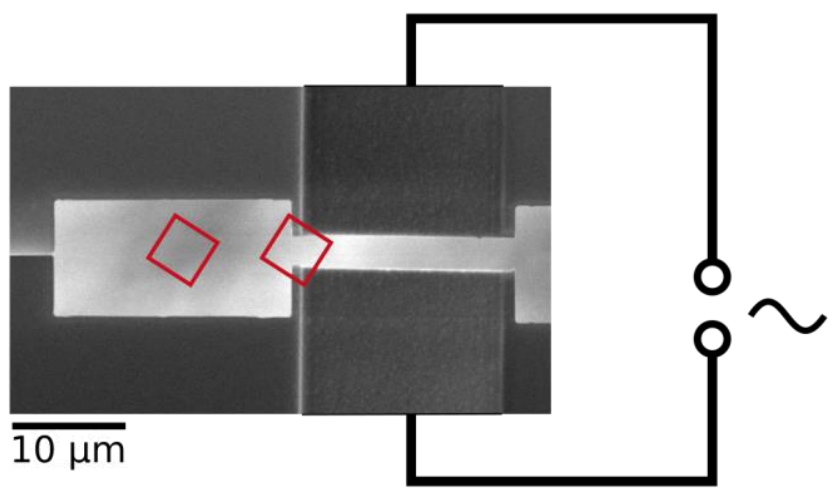

Figure 2. A severed metal wiring of a DENS heating chip is used as test specimen. The red rectangles mark the regions brought into overlapped by the electron optical biprism.
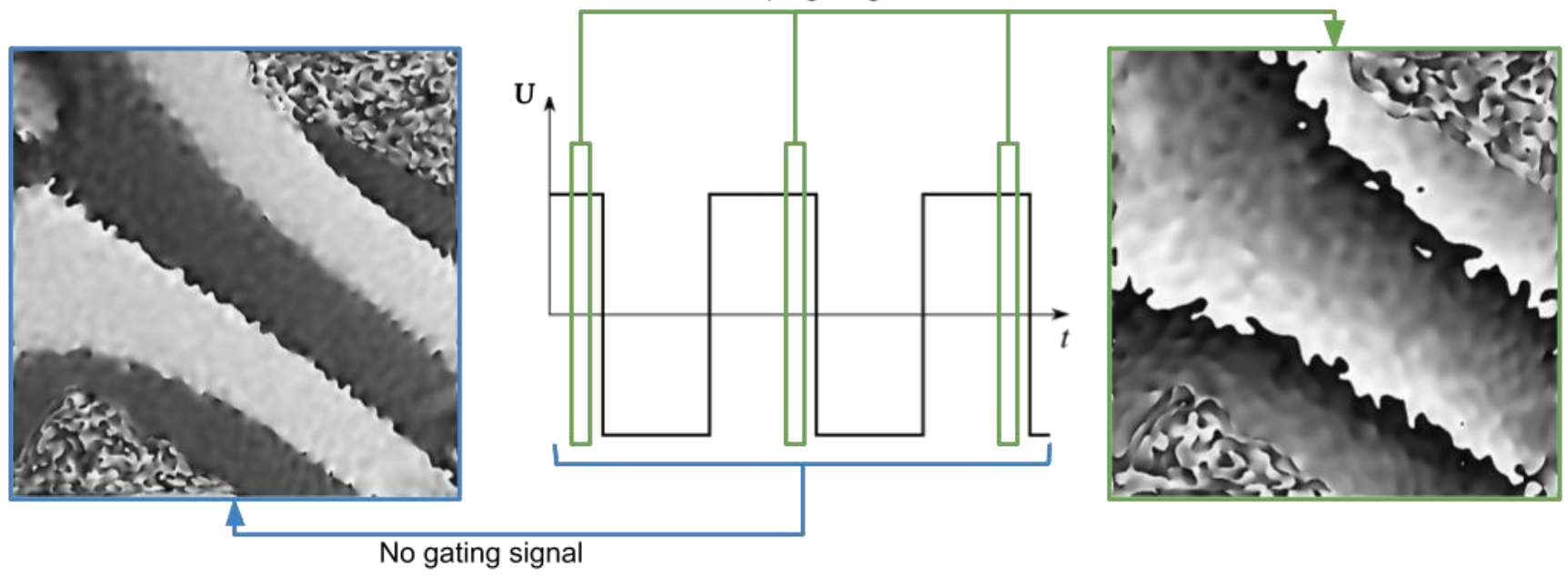

Figure 3. External voltage applied to the test specimen and reconstructed phases of two holograms. left: when no gating signal is present; right: with gating, the hologram is only obtained during the marked time intervals. 